\title{
Reporting National Outcomes After Esophagectomy and Gastrectomy According to the Esophageal Complications Consensus Group (ECCG)
}

\author{
Leonie R. van der Werf, MD, ${ }^{*}$ Linde A. D. Busweiler, MD, PhD, $\dagger$ Johanna W. van Sandick, MD, PhD, $\ddagger$ \\ Mark I. van Berge Henegouwen, MD, PhD, $\dagger$ and Bas P. L. Wijnhoven, MD, PhD*, the Dutch Upper GI Cancer \\ Audit (DUCA) group
}

\begin{abstract}
Objective: This nation-wide population-based study aimed to report postoperative morbidity and mortality after esophagectomy and gastrectomy in the Netherlands according to the definitions of the Esophagectomy Complications Consensus Group (ECCG).

Background: To standardize international outcome reporting in esophageal surgery, the ECCG developed a standardized outcomes set.

Methods: For this national cohort study, all patients undergoing esophagectomy or gastrectomy for cancer between 2016 and 2017 were selected from the Dutch Upper gastrointestinal Cancer Audit. In a random sample of hospitals, data completeness and accuracy were validated by reabstraction of the data. The investigated outcomes in the present study were postoperative complications, major complications (Clavien-Dindo grade $\geq$ III), and 30-day mortality, according to definitions of the ECCG.

Results: A total of 2545 patients from 22 hospitals were included. The completeness of the Dutch Upper gastrointestinal Cancer Audit was estimated at $99.8 \%$. Data accuracy on different items was $94 \%$ to $100 \%$. After esophagectomy, 1046 of 1617 patients (65\%) had a postoperative complication including 468 patients (29\%) with a major complication. Most common complications were pneumonia (21\%), esophago-enteric leak from anastomosis, staple line or localized conduit necrosis (19\%), and atrial dysrhythmia (15\%). The 30-day mortality was $1.7 \%$. After gastrectomy, 397 of 928 patients (42\%) had a postoperative complication including 180 patients (19\%) with a major complication. Most common complications were pneumonia (12\%), esophago-enteric leak from anastomosis, staple line or localized conduit necrosis (9\%), and acute delirium (5\%). The 30-day mortality was $4.4 \%$. Conclusions: Reporting complications according to the ECCG platform is feasible in the Netherlands and facilitates international benchmarking.
\end{abstract}

Keywords: clinical auditing, esophagectomy, esophagectomy complication consensus group, gastrectomy, outcomes-set

(Ann Surg 2020;271:1095-1101)

E or resectable nonmetastatic esophageal and gastric cancer, resec- tion is as yet the cornerstone of treatment. Both esophagectomy and gastrectomy are associated with high postoperative morbidity rates. To evaluate quality of care, in several European countries

From the *Erasmus University Medical Center, Rotterdam, The Netherlands; †Cancer Center Amsterdam, Amsterdam UMC, Amsterdam, The Netherlands; and $\ddagger$ Antoni van Leeuwenhoek Hospital, Amsterdam, The Netherlands.

The authors report no conflicts of interest.

Supplemental digital content is available for this article. Direct URL citations appear in the printed text and are provided in the HTML and PDF versions of this article on the journal's Web site (www.annalsofsurgery.com).

Reprints: Leonie R. van der Werf, MD, Erasmus University Medical Centre, 'Postbus 2040,3000 CA Rotterdam, The Netherlands.

E-mail: L.R.vdwerf@gmail.com.

Copyright (C) 2019 Wolters Kluwer Health, Inc. All rights reserved.

ISSN: 0003-4932/19/27106-1095

DOI: $10.1097 /$ SLA.0000000000003210 clinical audits are used. ${ }^{1-3}$ Feedback of audit data to the specialist may improve outcomes by stimulating best practices and the initiation of improvement programs for health care pathways. For a reliable comparison of outcomes between hospitals on a national level and to compare patterns of care and outcomes between countries, it is important to use uniform definitions.

To standardize outcome reporting in esophageal surgery, the Esophagectomy Complications Consensus Group (ECCG) developed a standardized outcomes-set. ${ }^{4}$ In 2017, in 24 hospitals in different countries the outcomes after esophagectomy were collected according to the definitions of the ECCG. ${ }^{5}$

In January 2016, the definitions of the ECCG were introduced in the Dutch Upper gastrointestinal Cancer Audit (DUCA). ${ }^{1}$ The outcomes including postoperative complications, readmission, and 30-day mortality were registered according to the definitions of the ECCG platform for both esophagectomy and gastrectomy. At that time, an international standardized outcomes-set for gastrectomy was lacking. Hence, the ECCG outcomes-set was applied for patients who underwent esophagectomy and gastrectomy also because the type and severity of complications that occur after both procedures is somewhat comparable.

The primary aim of this study was to report postoperative morbidity and mortality after esophagectomy and gastrectomy in the Netherlands according to the ECCG definitions and to report the completeness and accuracy of the DUCA data. Second, the outcomes after esophagectomy in the DUCA were compared with the reported outcomes of the initial ECCG dataset. ${ }^{5}$

\section{METHODS}

\section{Study Design}

For this national cohort study, patient data were retrieved from the DUCA database. Dutch hospitals are mandated to register all esophageal (including gastro-esophageal junction) or gastric cancer patients undergoing surgery with the intent of a resection.

\section{Data Verification}

Before evaluation of the DUCA data, it is important to test whether the outcomes are valid. The reliability of data of the data was verified in 2016. Participation of hospitals in this data verification process was voluntary. Outcomes of this data verification were the completeness and accuracy of registered data. A random sample of 15 participating hospitals was visited by an external data verification employee and a random sample of operated patients with esophageal or gastric cancer was checked for inclusion in the DUCA database. Per hospital, 30 patients operated in 2016 were selected. If less than 30 patients were operated, all available patients were selected. Reabstraction of data from the electronic 
patient dossier took place for all selected patients. The original data was compared with data registered in the DUCA. ${ }^{6}$ In the present study, the accuracy with regard to registration of postoperative complications, 30-day mortality, reinterventions, readmissions, number of lymph nodes, resections margin, and ASA score (the physical status classification according to the American Society of Anesthesiologists) was tested. The accuracy was estimated by the number of discrepancies found against the total number of patients in the sample.

\section{Patients}

All patients undergoing an esophagectomy of gastrectomy in the Netherlands between January 2016 and December 2017 for esophageal or gastric cancer were included in this study. Patients with a palliative bypass procedure were excluded. Also, patients with missing data regarding complications or other essential elements of the registration including date of birth, survival status at 30 days after surgery or date of discharge (in case of a hospital stay of $>30 \mathrm{~d}$ ) were excluded.

\section{Outcomes}

The primary outcome was frequency of postoperative complications. The severity of the complications was defined according to Clavien-Dindo. ${ }^{7}$ Complications grade IIIa or higher were defined as major complications. The secondary outcomes were hospital stay, duration of stay at the intensive care unit, the frequency of reinterventions, 30-day and/or in-hospital mortality, readmissions, the number of retrieved lymph nodes, surgical resection margins, and the ASA score. For all patients who underwent an esophagectomy, the outcomes were compared with the outcomes of the ECCG as recently reported. ${ }^{5}$

\section{Statistical Methods}

Patient and tumor characteristics of all included patients were reported according to the type of resection (esophagectomy or gastrectomy) using frequencies and percentages. Also, all postoperative outcomes were described using frequencies and percentages. The outcomes after esophagectomy in the DUCA were compared with the reported outcomes of the ECCG dataset ${ }^{5}$ using chi-square analyses. Statistical analyses of the present study were performed using Microsoft Excel for Mac (version 15.41). Statistical significance was defined as $P<0.05$.

\section{RESULTS}

\section{Data Verification}

The completeness of the DUCA was estimated at $99.8 \%$ (Table 1). In a sample of 408 patients, 1 patient who should have been registered according to the inclusion criteria of the DUCA was not registered. Complications were accurately registered in 382 of 407 patients (94\%). In 25 patients (6\%) no complication was registered in the DUCA, whereas in the electronical patient file a complication was reported. Thirty-day and/or in-hospital mortality was accurately registered in 406 of 407 patients (98.8\%). In 13 of 407 patients (3\%), a complicated postoperative course (defined as a complication leading to prolonged hospital stay $(>21 \mathrm{~d})$, reintervention or death) was not registered in the DUCA database but was extracted from the electronic patient files. All verified variables are shown in Table 1.

\section{Patients}

From January 2016 to December 2017, a total of 1617 patients undergoing an esophagectomy and 928 patients undergoing a gastrectomy were registered in the DUCA. Eight patients were excluded due to missing data. Patient, disease, and treatment characteristics are summarized in Tables 2 and 3. Minimally invasive techniques were used in $86 \%$ of patients undergoing an esophagectomy and in $58 \%$ of patients undergoing a gastrectomy. Fifty-two percent of esophagectomies was performed via a transthoracic approach. In $43 \%$ of all gastrectomies, a total gastrectomy was performed.

\section{Outcomes After Esophagectomy}

Sixty-five percent of patients who underwent an esophagectomy had a postoperative complication (Table 4). Clavien-Dindo grade III or higher complications occurred in $29 \%$ of all patients (Table 5). Most common complications were pneumonia (21\%), leak from the anastomosis, staple line or localized conduit necrosis (19\%), and atrial dysrhythmia (15\%). All complications are presented in Supplementary Table 1 (Supplemental Digital Content, http://links. lww.com/SLA/B561). The median stay at the intensive care unit was 2 days (interquartile range: 1-4), and median hospital stay was

TABLE 1. Results of External Data Verification

\begin{tabular}{|c|c|c|c|c|}
\hline \multicolumn{5}{|c|}{ Completeness of Data } \\
\hline & Registered & Wrongly Not $\operatorname{Re}$ & & Completeness \\
\hline Sample Size: 408 & $\mathbf{n}$ & $\mathbf{n}$ & & $\%$ \\
\hline Included in DUCA & 407 & 1 & & $99.8 \%$ \\
\hline \multicolumn{5}{|c|}{ Accuracy of Data } \\
\hline & Correctly Registered & Wrongly Registered & Missing & Accuracy \\
\hline Sample Size: 407 & $\mathbf{n}$ & $\mathbf{n}$ & $\mathbf{n}$ & $\%$ \\
\hline Complications & 382 & 25 & 0 & $94 \%$ \\
\hline 30-d/in-hospital mortality & 406 & 1 & 0 & $99.8 \%$ \\
\hline Reinterventions & 394 & 13 & 0 & $97 \%$ \\
\hline $\begin{array}{l}\text { Complications leading to } \\
\text { prolonged hospital stay } \\
(>21 \mathrm{~d}), \text { reintervention } \\
\text { or death }\end{array}$ & 394 & 13 & 0 & $97 \%$ \\
\hline Readmission & 390 & 12 & 5 & $97 \%$ \\
\hline Number of lymph nodes & 394 & 13 & 0 & $97 \%$ \\
\hline Resection margins & 394 & 11 & 2 & $97 \%$ \\
\hline ASA score & 379 & 28 & 0 & $93 \%$ \\
\hline
\end{tabular}


TABLE 2. Patient and Disease Characteristics, According to Type of Resection: Esophagectomy (for ECCG ${ }^{1}$ and DUCA) and Gastrectomy DUCA only Are Shown

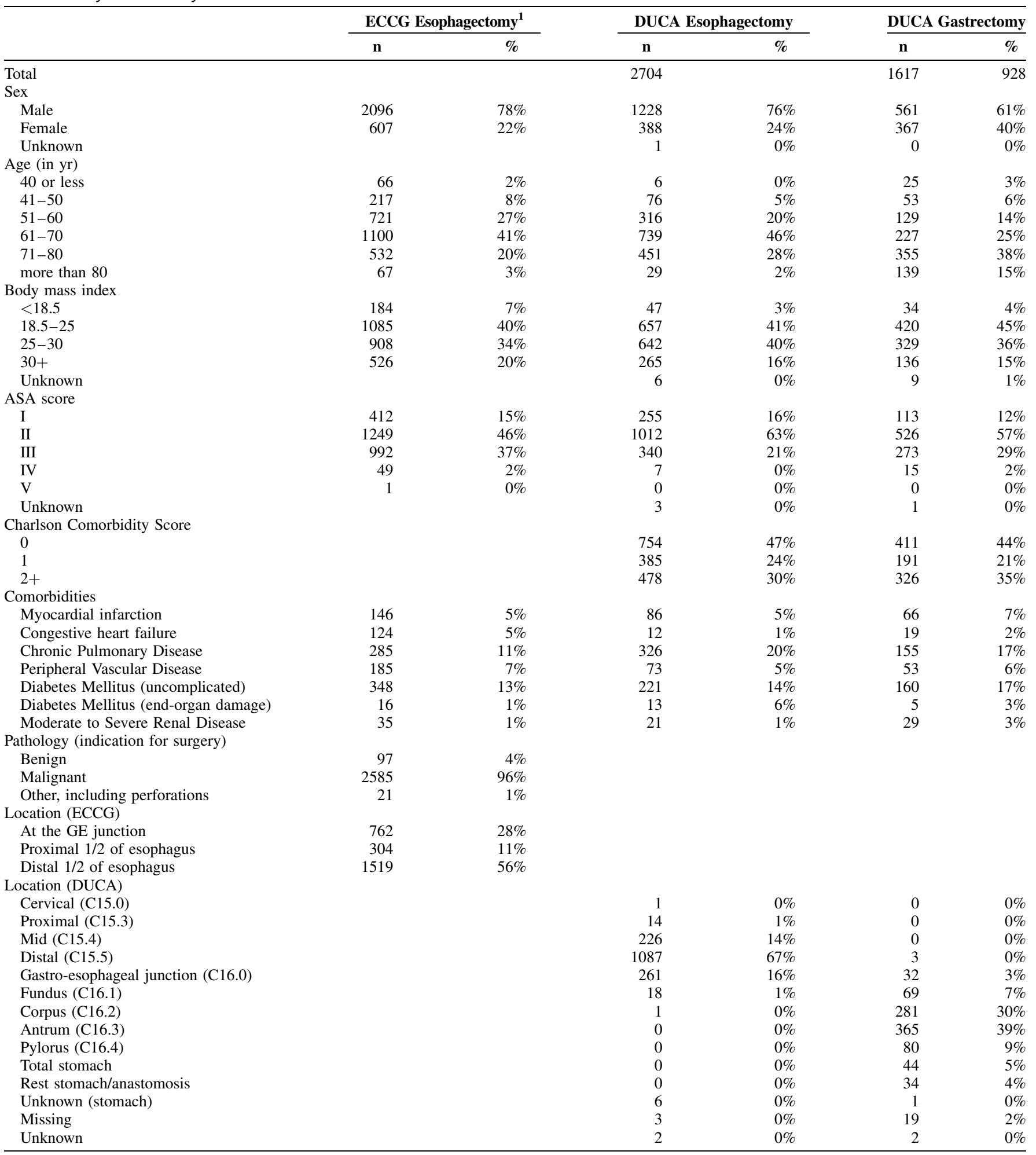

1. Low DE, Kuppusamy MK, Alderson D, et al. Benchmarking Complications Associated with Esophagectomy. Ann Surg 2017. ASA indicates American Society of Anaestesiologists. 
TABLE 3. Pathological- and Treatment Characteristics, According to Type or Resection: Esophagectomy (for ECCG ${ }^{1}$ and DUCA) and Gastrectomy DUCA only Are Shown

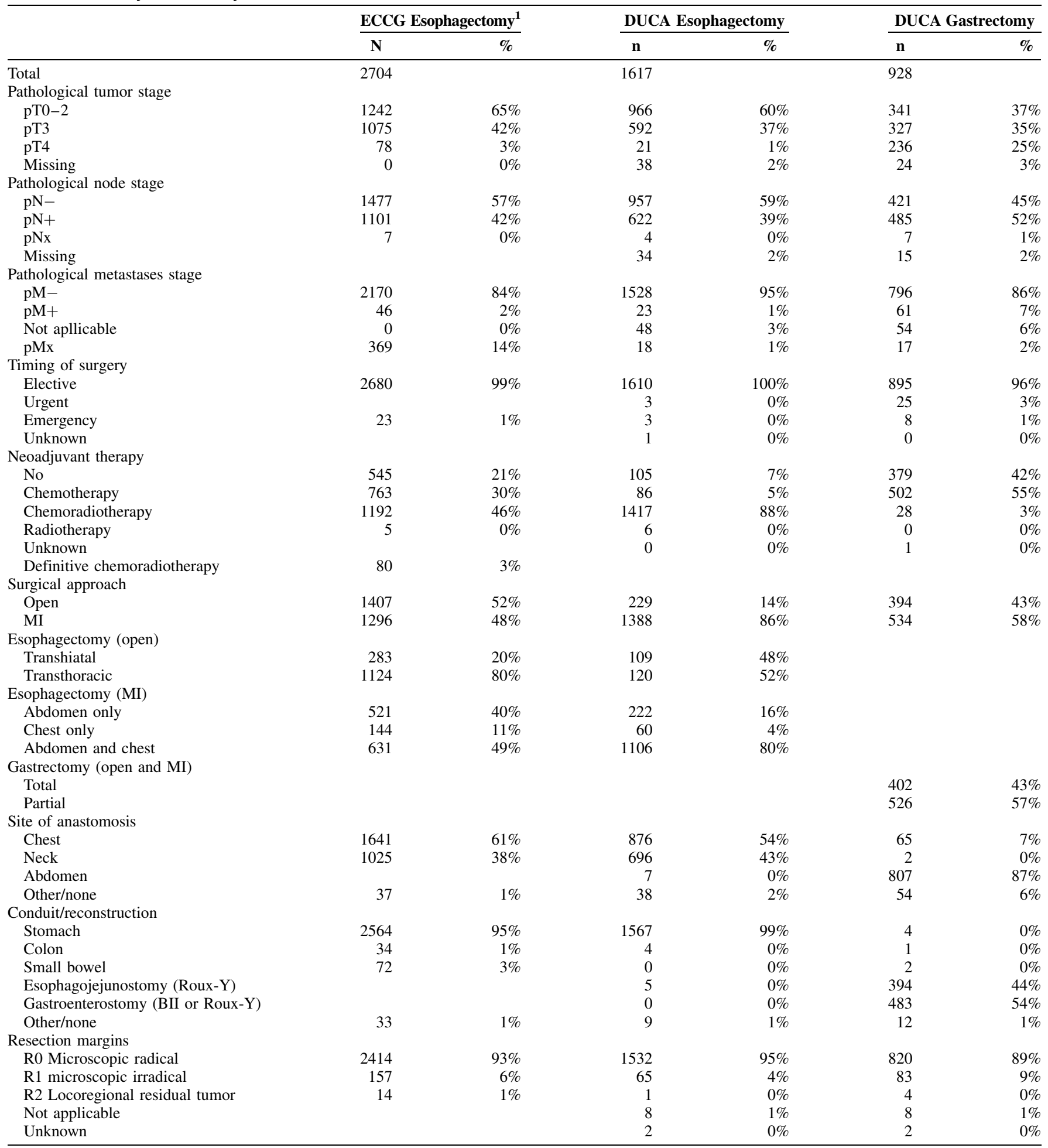

1. Low DE, Kuppusamy MK, Alderson D et al. Benchmarking Complications Associated with Esophagectomy. Ann Surg 2017.

MI indicates minimally invasive. 
TABLE 4. Outcomes of the DUCA (Dutch Upper Gastrointestinal Cancer Audit) According to Type of Resection

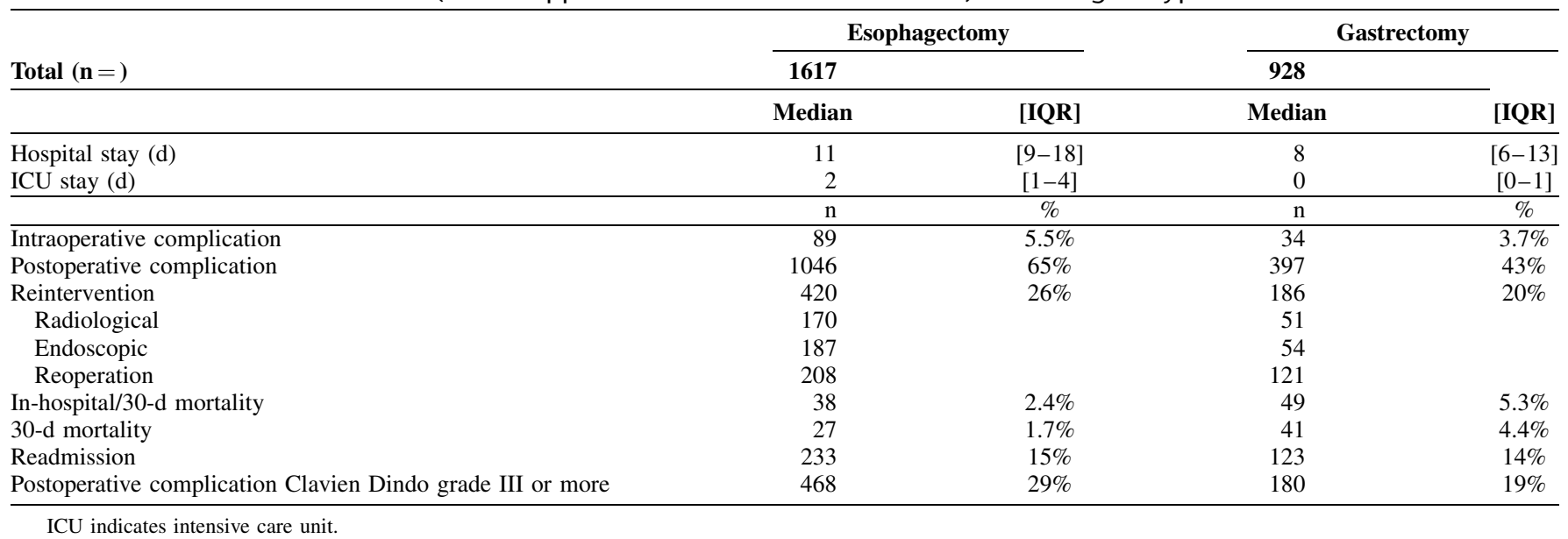

TABLE 5. Severity of Complications in the DUCA (Dutch Upper Gastrointestinal Cancer Audit) According to Type of Resection

\begin{tabular}{|c|c|c|c|c|c|c|c|c|}
\hline \multirow[b]{2}{*}{ Complication Severity } & \multicolumn{4}{|c|}{ Esophagectomy } & \multicolumn{4}{|c|}{ Gastrectomy } \\
\hline & $\mathbf{n}$ & $\%$ & $95 \%$ & CI & $\mathbf{n}$ & $\%$ & $\mathbf{9 5 \%}$ & CI \\
\hline No complications & 605 & $37 \%$ & $35 \%$ & $40 \%$ & 562 & $61 \%$ & $57 \%$ & $64 \%$ \\
\hline Grade I & 150 & $9 \%$ & $8 \%$ & $11 \%$ & 39 & $4 \%$ & $3 \%$ & $6 \%$ \\
\hline Grade IIIa & 192 & $12 \%$ & $10 \%$ & $14 \%$ & 51 & $6 \%$ & $4 \%$ & $7 \%$ \\
\hline Grade IIIb & 128 & $8 \%$ & $7 \%$ & $9 \%$ & 66 & $7 \%$ & $6 \%$ & $9 \%$ \\
\hline Grade IVa & 110 & $7 \%$ & $6 \%$ & $8 \%$ & 23 & $3 \%$ & $2 \%$ & $4 \%$ \\
\hline Grade unknown & 15 & $1 \%$ & $1 \%$ & $2 \%$ & 17 & $2 \%$ & $1 \%$ & $3 \%$ \\
\hline
\end{tabular}

11 days (interquartile range: $9-18$ ). The 30-day mortality rate was $1.7 \%$ and the 30 -day/in-hospital mortality rate was $2.4 \%$.

In comparison with the reported outcomes of the ECCG,${ }^{5}$ the overall complication rate was significantly higher in the DUCA $(65 \%$ vs $59 \%, P<0.001)$. Also, pneumonia and leak from anastomosis, staple line, or localized conduit necrosis, occurred more often (respectively, $21 \%$ vs $15 \%, P<0.001$ and $19 \%$ vs $11 \%, P<$ 0.001 ) (Fig. 1). Hospital readmission within 30 days after discharge

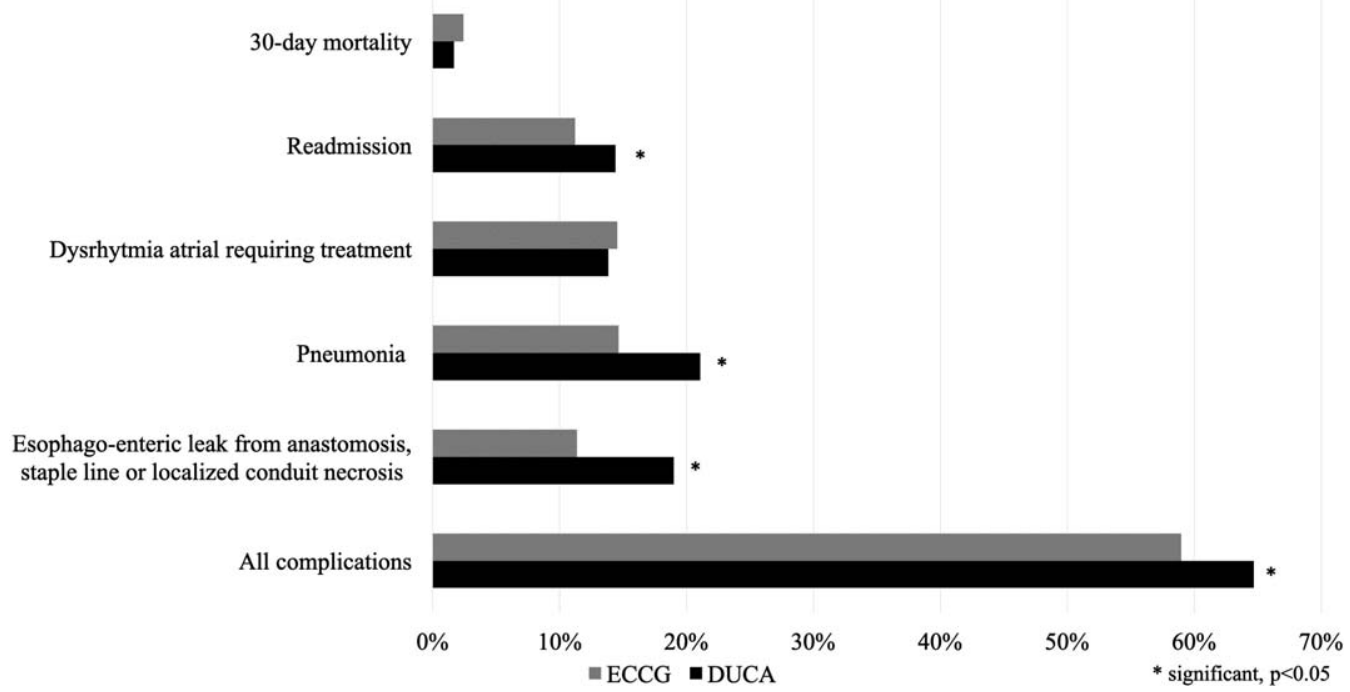

FIGURE 1. Comparison of outcomes after esophagectomies, DUCA versus ECCG. 
occurred in $15 \%$ of patients, significantly more often compared with the ECCG cohort $(11 \%, P<0.001)$. The 30 -day mortality rate was $1.7 \%$ versus $2.4 \%$ in the ECCG cohort $(P=0.10)$.

\section{Outcomes After Gastrectomy}

Forty-three percent of patients who underwent a gastrectomy experienced a postoperative complication (Table 4). Clavien-Dindo grade III or higher complications occurred in $19 \%$ of patients (Table 5). Most common complications were pneumonia (12\%), esophago-enteric leak from anastomosis, staple line or localized conduit necrosis (9\%), and acute delirium (5\%). All complications are presented in Supplementary Table 1 (Supplemental Digital Content, http://links.lww.com/SLA/B561). The severity of 4 outcome measures according to the $\mathrm{ECCG}^{4}$ is presented in Supplementary Table 2, Supplemental Digital Content, http://links.lww.com/SLA/B561. The median stay at the intensive care unit stay was 0 days (interquartile range: $0-1$ ), and median hospital stay was 8 days (interquartile range: 6-13). Hospital readmission within 30 days after discharge occurred in $14 \%$ of patients. The 30 -day mortality rate was $4.4 \%$, and the 30 day/in-hospital mortality was $5.3 \%$.

\section{DISCUSSION}

This study with DUCA data shows that reporting complications according to the ECCG definitions can be achieved on a national level. Data verification showed that the completeness and accuracy of data in the DUCA were high. Overall, complications after esophagectomy and gastrectomy occurred in $65 \%$ and $43 \%$ of patients, respectively. Major complications (Clavien-Dindo grade III or higher) occurred in $29 \%$ and $19 \%$ of patients, respectively. The most common complications after esophagectomy were pneumonia, esophago-enteric leak, and dysrhythmia atrial. After gastrectomy, pneumonia, esophago-enteric leak, and acute delirium were the most common complications.

Recently, the outcomes of 24 high volume hospitals participating in the ECCG were published. ${ }^{5}$ Compared with these data, overall complication rates, pneumonia rates, and esophago-enteric leakage rates were significantly higher in the DUCA. Different explanations may exist for these discrepancies.

First, differences in patient and treatment characteristics exist between the ECCG cohort and DUCA cohort which might have influenced the occurrence of complications. From previous studies with DUCA data, it is known that higher age, ASA score, body mass index, $\mathrm{N}+$ status, proximal-mid esophageal tumorlocation, and open transthoracic procedures are associated with an increased risk for postoperative complications. ${ }^{8,9}$ Some of these factors were more frequently present in the DUCA, for example, $30 \%$ of patients were older than 70 years old (vs $23 \%$ in the ECCG cohort). However, in the ECCG cohort, patients with ASA III or higher were more frequently present then in the DUCA (39\% vs $21 \%)$.

The second difference was the percentage of patients that was treated with neoadjuvant chemoradiotherapy. In the DUCA, $88 \%$ of patients were treated with neoadjuvant chemoradiotherapy, versus $46 \%$ in the ECCG cohort. In the literature, some studies regarding neoadjuvant chemoradiotherapy have reported no significant differences in complication rates between neoadjuvant chemoradiotherapy and neoadjuvant chemotherapy alone or no neoadjuvant therapy. ${ }^{10-12}$ However, Klevebro et al ${ }^{12}$ reported a higher frequency of severe complications after neoadjuvant chemoradiotherapy in comparison with neoadjuvant chemotherapy alone. It has been suggested that radiotherapy affects the lung tissue and may increase pulmonary complications. ${ }^{13}$ The difference in type and frequency of neoadjuvant therapy could be an explanation of the higher pneumonia rate in the DUCA versus the ECCG cohort. A study with combined datasets and correction for differences in case-mix could potentially answer this issue.

Another difference between both cohorts was the type of esophagectomy. In the DUCA $86 \%$ of patients underwent a minimally invasive esophagectomy versus $48 \%$ in the ECCG. The TIME trial, a randomized trial evaluating minimally invasive versus open transthoracic esophagectomy, showed that in-hospital pulmonary infections occurred significantly less frequent after minimally invasive esophagectomy (12\% vs $34 \%) .{ }^{14}$ A previous Dutch study showed that during the implementation of minimally invasive esophagectomies in the Netherlands there were no differences in pulmonary complications and 30-day/in-hospital mortality between minimally invasive versus open gastrectomy. However, the same study showed higher anastomotic leakage rates and reintervention rates after minimally invasive gastrectomy. ${ }^{15}$ The introduction of minimally invasive surgery and the associated learning curve that goes with it, ${ }^{16}$ might have influenced the complication rate. Nonetheless, in $2015,84 \%$ of the registered esophagectomies in the DUCA was performed with minimally invasive techniques and, since the current study only reports data of 2016 and 2017, it could be that most surgeons might already have completed their learning curve in this period. However, it is important to keep in mind that learning curve until proficiency might be much longer than initially was expected. ${ }^{16}$ Future studies are needed to evaluate the "real" length of the learning curve. Also the approach of esophagectomy differed between the DUCA and ECCG cohorts. The transhiatal approach was more favourite in the DUCA cohort than in the ECCG cohort: $48 \%$ versus $20 \%$, respectively. As reported in a meta-analysis of Hulscher et al, ${ }^{17}$ the transhiatal approach and cervical anastomosis is associated with a higher frequency of anastomotic leakage and vocal cord paralysis. In the transthoracic group in this meta-analysis, there was more perioperative blood loss, pulmonary complications, chyle leak, and wound infections. Thus, the difference in favored approaches between the DUCA and ECCG might explain the higher anastomotic leakage rate in the DUCA database. Nonetheless, the higher pneumonia rate in the DUCA could not be explained by the differences in surgical approach.

The annual hospital volume of the participating hospitals in the ECCG has been described as all "high volume." In the DUCA, in 2016, the annual hospital volume varied each year. In 2016, 9 of 22 hospitals performed 40 or more resections and 5 hospitals performed less than 20 resections. ${ }^{18}$ Differences in annual hospital volume may influence outcomes. However, further studies are needed to evaluate whether these differences can explain the variation in outcomes between the cohorts.

Due to the use of a standardized outcomes set, the DUCA outcomes after esophagectomies could be compared with the ECCG outcomes fairly. For outcomes after gastrectomies, at the time of the implementation of the ECCG outcomes, there was no standardized international consensus set and the ECCG outcomes were also incorporated for patients after gastrectomy. To our knowledge, the ECCG outcomes set has not been used for reporting outcomes after gastrectomy in other cohorts. Recently, a specific standardized outcomes set for gastric cancer surgery was published with the intent to facilitate international comparison. ${ }^{19}$ The intent is to implement this standardized set of definitions in the DUCA because it potentially facilitates international comparison.

An international comparison of Dutch results after esophagectomy and gastrectomy has been done previously. The results of the DUCA were compared with the results of the Swedish NREV (Nationellt Kvalitetsregister matstrups-och magsäckscancer). ${ }^{20}$ However, the results of the registries at that time were not standardized, which makes comparison not really reliable. 
The 30-day mortality in the DUCA database was $1.7 \%$ after esophagectomy and $4.4 \%$ after gastrectomy. In comparison with the outcomes of the ECCG cohort, the mortality after esophagectomies was not significantly different. The 30-day mortality after gastrectomy and esophagectomy was also reported in the annual report of the British "National Oesophago Gastric Cancer Audit." Between 2007 and 2009 and between 2013 and 2015, the 30-day mortality after esophagectomies was $3.8 \%$ (95\% confidence interval: $3.1 \%-4.7 \%$ ) and $1.6 \%$ (95\% confidence interval: $1.2 \%-$ $2.1 \%$ ), respectively. After gastrectomy it was $4.5 \%$ (95\% confidence interval: $3.4 \%-5.7 \%$ ) and $1.9 \%$ (95\% confidence interval $1.3 \%-2.7 \%$ ), respectively. In the annual report of the National Oesophago Gastric Cancer Audit, no clarification was given for this improvement in 30-day mortality after esophagectomy and gastrectomy. It would be interesting to evaluate the underlying processes; to direct a strategy to also improve 30-day mortality after gastrectomy in the Netherlands.

In conclusion, evaluation of quality of care is important, especially for high complex, low-volume procedures such as esophagectomy and gastrectomy. Reporting outcomes using standardized definitions is an essential step toward reliable results. Furthermore, it enables international comparisons that could help to reveal significant differences in outcomes and to identify factors which could be improved. A more widespread adoption of the ECCG platform could be recommended to improve international benchmarking in esophageal surgery.

\section{ACKNOWLEDGMENTS}

The authors thank all surgeons, registrars, physician assistants, and administrative nurses for data registration in the DUCA database, as well as the Dutch Upper GI Cancer Audit group for scientific input.

Collaborators: The following members of the DUCA group were collaborators in this study: K. Bosscha (Department of Surgery, Jeroen Bosch Hospital, 's-Hertogenbosch), A. Cats, (Department of Gastroenterology, the Netherlands Cancer Institute - Antoni van Leeuwenhoek Hospital, Amsterdam), J.L. Dikken (Department of Surgery, Leiden University Medical Centre), N.C.T. van Grieken (Department of Pathology, VU University Medical Centre, Amsterdam), H.H. Hartgrink (Department of Surgery, Leiden University Medical Centre, Leiden), R. van Hillegersberg (Department of Surgery, University Medical Centre Utrecht, Utrecht), V.E.P.P. Lemmens (Department of Epidemiology, Erasmus University Medical Centre, Rotterdam, IKNL), G.A.P. Nieuwenhuijzen (Department of Surgery, Catharina Hospital, Eindhoven), J.T. Plukker (Department of Surgery, University Medical Centre Groningen, Groningen), C. Rosman (Department of Surgery, Radboud University Medical Centre, Nijmegen), P.D. Siersema (Department of Gastroenterology and Hepatology, Radboud University Medical Centre, Nijmegen), G. Tetteroo (Department of Surgery, IJsselland Ziekenhuis, Capelle a/d IJssel), P.M.J.F. Veldhuis (Department of Oncological Care, IKNL), F.E.M. Voncken (Department of radiotherapy, the Netherlands Cancer Institute - Antoni van Leeuwenhoek Hospital, Amsterdam).

\section{REFERENCES}

1. Busweiler LA, Wijnhoven BP, van Berge Henegouwen MI, et al. Early outcomes from the Dutch Upper Gastrointestinal Cancer Audit. Br J Surg. 2016;103:1855-1863.

2. Clinical Effectiveness Unit TRCoSoE. National Oesophago-Gastric Cancer Audit 2016. In An audit of the care received by people with OesophagoGastric Cancer in England and Wales 2016 Annual Report. 2016.

3. Emilsson L, Lindahl B, Koster M, et al. Review of 103 Swedish Healthcare Quality Registries. J Intern Med. 2015;277:94-136.

4. Low DE, Alderson D, Cecconello I, et al. International consensus on standardization of data collection for complications associated with esophagectomy: esophagectomy complications consensus group (ECCG). Ann Surg. 2015;262:286-294.

5. Low DE, Kuppusamy MK, Alderson D, et al., Benchmarking Complications Associated with Esophagectomy. Benchmarking complications associated with esophagectomy. Ann Surg. 2019;269:291-298.

6. van der Werf LR, Voeten SV, van Loe CA, et al. Data verification of national clinical audits in the Netherlands. Under review.

7. Dindo D, Demartines N, Clavien PA. Classification of surgical complications: a new proposal with evaluation in a cohort of 6336 patients and results of a survey. Ann Surg. 2004;240:205-213.

8. van der Werf LR, Dikken JL, van der Willik EM, et al. Time interval between neoadjuvant chemoradiotherapy and surgery for oesophageal or junctional cancer: a nationwide study. Eur J Cancer. 2018;91:76-85.

9. van der Werf LR, Dikken JL, van Berge Henegouwen MI, et al. A populationbased study on lymph node retrieval in patients with esophageal cancer: results from the dutch upper gastrointestinal cancer audit. Ann Surg Oncol. 2018;25:1211-1220.

10. van Hagen P, Hulshof MC, van Lanschot JJ, et al. Preoperative chemoradiotherapy for esophageal or junctional cancer. N Engl J Med. 2012;366:2074-2084.

11. Burmeister $\mathrm{BH}$, Thomas JM, Burmeister EA, et al. Is concurrent radiation therapy required in patients receiving preoperative chemotherapy for adenocarcinoma of the oesophagus? A randomised phase II trial. Eur J Cancer. 2011;47:354-360.

12. Klevebro F, Johnsen G, Johnson E, et al. Morbidity and mortality after surgery for cancer of the oesophagus and gastro-oesophageal junction: a randomized clinical trial of neoadjuvant chemotherapy vs. neoadjuvant chemoradiation. Eur J Surg Oncol. 2015;41:920-926.

13. Sathornviriyapong S, Matsuda A, Miyashita M, et al. Impact of neoadjuvant chemoradiation on short-term outcomes for esophageal squamous cell carcinoma patients: a meta-analysis. Ann Surg Oncol. 2016;23:3632-3640.

14. Biere SS, van Berge Henegouwen MI, Maas KW, et al. Minimally invasive versus open oesophagectomy for patients with oesophageal cancer: a multicentre, open-label, randomised controlled trial. Lancet. 2012;379:1887-1892.

15. Seesing MFJ, Gisbertz SS, Goense L, et al. A propensity score matched analysis of open versus minimally invasive transthoracic esophagectomy in the Netherlands. Ann Surg. 2017;266:839-846.

16. van Workum F, Stenstra MHBC, Berkelmans GHK, et al. Learning curve and associated morbidity of minimally invasive esophagectomy: a retrospective multicenter study. Ann Surg. 2019;269:88-94.

17. Hulscher JB, Tijssen JG, Obertop H, et al. Transthoracic versus transhiatal resection for carcinoma of the esophagus: a meta-analysis. Ann Thorac Surg. 2001;72:306-313.

18. Dutch Institute for Clinical Audits. TOEGENOMEN AANTAL MAAG-EN SLOKDARMRESECTIES PER ZIEKENHUIS (translation: Increased number of gastric and esophageal resections per hospital); 2017. https://dica.nl/ nieuws/duca-juni. Accessed January 15, 2019.

19. Baiocchi GL, Giacopuzzi S, Marrelli D, et al. International consensus on a complications list after gastrectomy for cancer. Gastric Cancer. 2019;22:172-189.

20. Busweiler LAD, Jeremiasen M, Wijnhoven BPL, et al. International benchmarking in oesophageal and gastric cancer surgery. BJS Open. 2018. doi: $10.1002 /$ bjs 5.50107 . 